\title{
Application of a new drainage plug for large mandibular cysts after fenestration decompression
}

\author{
Lin Zhang ${ }^{1,2}$, Jing Pei ${ }^{2,3}$, Min Huang ${ }^{1,2}$, Wenwei Lian ${ }^{1,2}$ \\ ${ }^{1}$ Department of Prosthodontics, The Affiliated Stomatological Hospital of Nanchang University, Nanchang, China; ${ }^{2}$ The Key Laboratory of Oral \\ Biomedicine, Nanchang, China; ${ }^{3}$ Department of Oral Pathology, The Affiliated Stomatological Hospital of Nanchang University, Nanchang, China \\ Contributions: (I) Conception and design: L Zhang; (II) Administrative support: W Lian; (III) Provision of study materials or patients: L Zhang; (IV) \\ Collection and assembly of data: M Huang; (V) Data analysis and interpretation: J Pei; (VI) Manuscript writing: All authors; (VII) Final approval of \\ manuscript: All authors. \\ Correspondence to: Wenwei Lian. Department of Prosthodontics, The Affiliated Stomatological Hospital of Nanchang University; The Key \\ Laboratory of Oral Biomedicine of Jiangxi Province, Nanchang 330006, China. Email: Lianwenwei1967@126.com.
}

\begin{abstract}
Background In order to improve the postoperative decompression and drainage of large mandibular cysts after fenestration decompression, a new drainage plug was designed and its feasibility for clinical application was explored.
\end{abstract}

Methods: A total of 74 patients with large mandibular cysts requiring fenestration decompression were included and randomly divided into the control group $(n=34)$ and model group $(n=40)$. Patients in the control group were given a conventional plug, while patients in the model group were given the new silicone drainage plug. The drainage plug mold was printed using 3D printing technology. Subsequently, the mold was filled with silicone material and the drainage tube was placed into the mold to make a drainage plug. The clinical effect of the new drainage plug was assessed, and the postoperative recovery time was compared between the 2 groups.

Results: In the model group, the average wear time of the new drainage plug was approximately 13 months. Compared with the control group, the course of treatment in the model group was shortened by approximately 5 months, with a better fit, less food debris, and easier installation and removal.

Conclusions: The new drainage plug provides more convenience and better prognosis for patients after fenestration decompression, and holds great promise for clinical application.

Keywords: Large mandibular cyst; fenestration decompression; new drainage plug; prognosis

Submitted Nov 27, 2020. Accepted for publication Jan 11, 2021.

doi: 10.21037/apm-20-2464

View this article at: http://dx.doi.org/10.21037/apm-20-2464

\section{Introduction}

Mandibular cyst, also known as mandibular cystic lesion, is one of the most common diseases of the oral and maxillofacial region. The disease is characterized by cystic masses containing fluid in the mandible, and is usually treated with surgery. Because early mandibular cysts have no obvious symptoms, it is difficult for patients to be diagnosed in the early stage (1). Patients are usually diagnosed after imaging due to facial deformities or other symptoms. According to cyst size, mandibular cysts can be classified into small, median, and large mandibular cysts, which often invade teeth and can seriously affect quality of life (2). Small and median mandibular cysts can generally achieve satisfactory results after root canal therapy (3), while curettage is an effective and radical treatment for median and large mandibular cysts (4). However, large mandibular cysts ( $>4 \mathrm{~cm}$ in diameter) lead to a wider range of involvement and more significant anatomical damage, and are more likely to cause problems such as bone destruction, 
maxillofacial deformation, and can affect occlusal function. Thus, the efficacy of conventional cyst curettage is not satisfactory (5-8).

Fenestration decompression, also known as fenestration drainage or marsupialization, is a conservative treatment for cysts which has been widely used in recent years (9-11). It mainly involves fenestration surgery to form an opening on the surface of the mouth close to the cyst. After that, a communication is formed between the cyst cavity and the oral or nasal cavity, leading to the timely drainage of effusion in the cyst. When there is no effusion pressure in the cyst cavity, bone tissues of the jaw around the cyst can partially reconstruct themselves, so the cyst cavity gradually becomes smaller to some extent $(12,13)$. However, the placement of a filler (plug) is immediately required after fenestration decompression to avoid wound deformity and food accumulation $(4,14)$.

Postoperative nursing of patients, such as continuous drainage and holding of the wound, leads to the need for more time and medical resources. Therefore, in this study, we designed a silicone resin-covered plug with drainage function. This drainage plug was made of an acrylic resin frame covered by a thin layer of silicone. The frame was constructed to adapt to individual patients based on the $\mathrm{X}$-ray scan results of the cyst and its surrounding environment (15). The drainage function of the plug was achieved by a venous tube $(2.5 \mathrm{~mm}$ in inner diameter $)$ embedded in the frame. Each plug was specifically designed according to the shape and length of the wound to the cyst wall. The drainage tube directly reached the root of the cyst, making it convenient to rinse the cyst using various oral lotions. In addition, the clinical effects of a conventional plug and this new drainage plug were compared, so as to assess the clinical application value of the new one.

We present the following article in accordance with the CONSORT reporting checklist (available at http://dx.doi. org/10.21037/apm-20-2464).

\section{Methods}

\section{Study subjects}

A total of 74 patients with mandibular cysts requiring fenestration decompression in our hospital between January 2013 and December 2018 were included. All procedures performed in this study involving human participants were in accordance with the Declaration of Helsinki (as revised in 2013). This study was approved by the Ethics
Committee of the Affiliated Stomatological Hospital of Nanchang University (No. 2019017). All study subjects were divided into the control group $(n=34)$ and the model group $(n=40)$ using a random number table. After fenestration decompression, patients in the control group wore conventional plugs, while patients in the model group wore new silicone resin-covered drainage plugs. Basic information of patients was collected, including gender, age, lesion location, lesion size, cyst type, and wear time of the plug.

\section{Screening criteria}

The inclusion criteria were as follows: (I) the lesion was located in the mandible; (II) patients had no history of jaw cysts; (III) patients who cooperated with follow-up; (IV) all patients volunteered to participate and provided informed consent. The exclusion criteria were as follows: (I) patients with surgical contraindications; (II) patients who did not cooperate with follow-up.

\section{Design and construction of the new drainage plug}

First, for the preparation of the drainage plug mold, the range and shape of the cyst cavity were determined using a panoramic $\mathrm{X}$-ray, and the range of cyst invasion was analyzed in $3 \mathrm{D}(16)$. The spatial structure of the cyst cavity was reconstructed using 3D printing (Figure 1A,B). The plug mold, which was adapted to the cyst cavity, was constructed in combination with $3 \mathrm{D}$ printing technology (17).

Second, to create the drainage plug, PMMA acrylic resin was first mixed with its solution (Nissin Dental Products Co., Ltd., Kyoto, Japan) to keep it in a semi-solidified state with flow property. Subsequently, the acrylic resin in its semi-solidified state was wrapped around a drainage tube with a diameter of $2.5 \mathrm{~mm}$ (Shanghai LUK Co., Ltd., China), and was then perfused into the plug mold to make a frame (Figure $2 A$ ). The length of each drainage tube was specifically customized according to the distance from the base of the cyst to the open end of the drainage plug. If the acrylic resin in a semi-solidified state fit poorly with the mold, adjustment was necessary to achieve a complete fit between the frame and the mold by using a tungsten steel burr (Suzhou Syndent Tools Co., Ltd., China).

Third, for the adaptation of the drainage plug, the frame was disinfected with $75 \%$ ethanol solution and dried for $1-$ 3 minutes after the PMMA acrylic resin frame had solidified. Silagum-Comfort primer was then applied (DMG, 

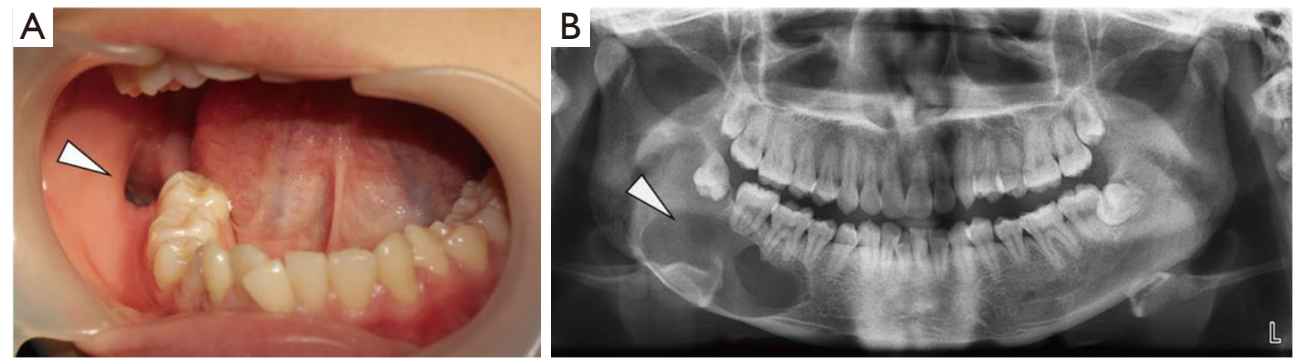

Figure 1 Odontogenic keratocyst. (A) The cyst cavity was located on the right side of the jaw (white arrow). (B) The location and size of the cyst is shown using a panoramic $\mathrm{X}$-ray scan (white arrow).
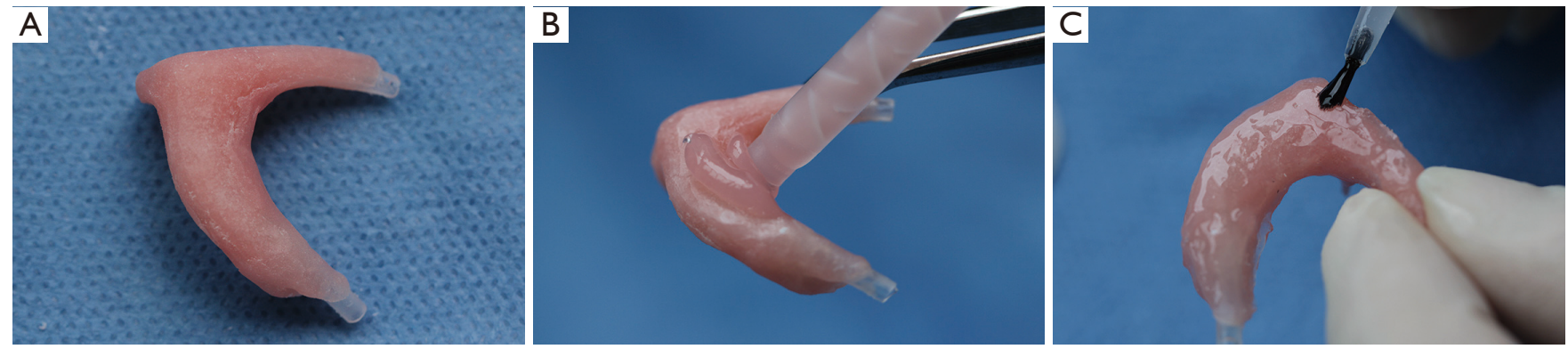

Figure 2 The clinical chairside manufacturing process of the drainage plug. (A) The frame was made of acrylic resin, and the transparent drainage tube was embedded in the frame. (B) The silicone soft lining was applied on the surface of the acrylic resin frame to completely fit the patient's mouth. (C) The surface of the silicone soft lining was covered with a layer of Silagum adaptive film.

Germany) and left to stand for 1 minute. Subsequently, the silicone soft lining was covered on the surface of the frame (Figure $2 B$ ), and the entire drainage plug was placed in the resulting cavity for 6 minutes until the silicone material was attached. Finally, the drainage plug was trimmed and covered with the Silagum adaptive film (Figure 2C).

\section{Clinical effect evaluation}

Patients in the model group wore the drainage plug, which was taken out after eating every day, then the cyst cavity was washed with oral lotion 2-3 times. The patients were followed up after 3, 6, 12, and 18 months, and the drainage plug was adjusted according to changes in the cyst cavity. The recovery time of patients in the control group and the model group was recorded.

Efficacy evaluation was based on the subjective evaluation of all patients. The evaluation items included retention of the plug, degree of comfort, deformation of the plug, installation and removal of the plug, oral odor, and impact on daily life. The evaluation criteria were as follows: (I) satisfactory: good retention, very comfortable, no deformation, easy removal, no tenderness and stimulation pain, no obvious oral odor, and no impact on daily life; (II) unsatisfactory: easy to loosen, with deformation, with obvious tenderness and stimulation pain on soft tissue during installation and removal, with obvious oral odor, with significant impact on daily life.

In addition, imaging measurements were performed. All patients underwent panoramic X-ray scanning at each return visit in order to calculate the size of the cyst area and observe the amount of new bone formation. Reduction rate of cyst area $=$ (preoperative cyst area - cyst area at return visit)/preoperative cyst area.

\section{Statistical analysis}

SPSS 25.0 was used for statistical analysis. Measurement data were expressed as mean \pm standard deviation (SD). Differences between the 2 groups were compared using a $t$-test. The Pearson correlation method was performed to analyze the correlation between cyst lesion size and treatment time. $\mathrm{P}<0.05$ was considered statistically significant. 


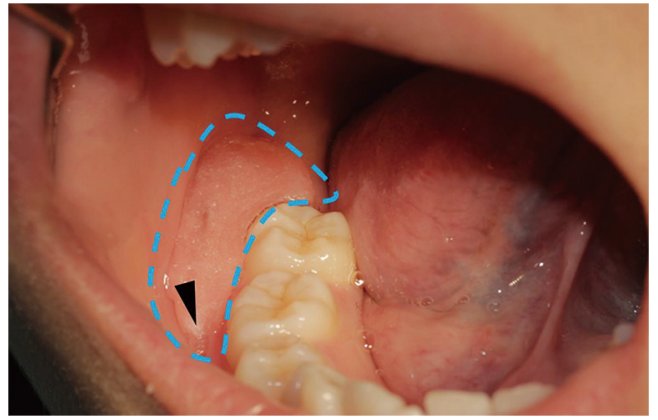

Figure 3 Schematic diagram of the installation of the drainage plug (blue circle). The blue circle represents the retention part (RP) of the drainage plug, which fit well with the surrounding environment of the mouth. The drainage tube opened lateral to the gum (black arrow), in order to facilitate timely drainage of the cyst effusion.

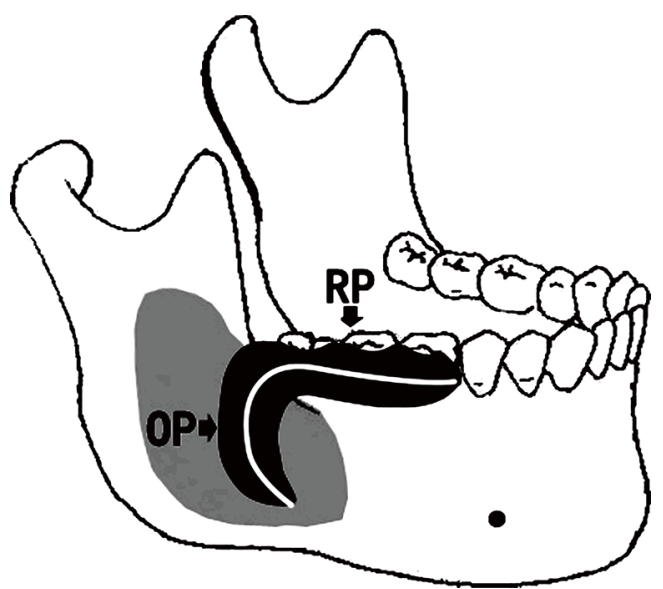

Figure 4 Pattern diagram of the drainage plug. The drainage plug contains two important parts: a retention part (PR), and an obturation part (OP).

\section{Results}

\section{Application and effect of the drainage plug}

In the model group, the drainage plug completely fitted the postoperative cavity without gaps (Figure 3). One end of the drainage tube passed straight into the cyst cavity [Figure 4, obturation part (OP)], and the other end opened in the mouth lateral to the gum [Figure 4, retention part (RP)] to ensure the timely drainage of cyst effusion. With the drainage plug, the recovery time of patients was effectively shortened, and the postoperative care time was also greatly reduced.

\section{New drainage plug shortens the recovery time of patients}

In the efficacy evaluation of the new drainage plug, the data of patients with different lesion degrees were collected. In general, patients with larger lesions were required to wear the plug for a longer time, and there was an approximately linear positive relationship between the two (Figure $5 A$ ). Wear time was significantly longer in the control group $(17.9 \pm 3.7$, average: approximately 18 months) than in the model group (13.3 \pm 2.9 , average: approximately 13 months) $(\mathrm{P}<0.0001)$. The new drainage plug reduced the treatment time by nearly 5 months, indicating significant efficacy (Figure 5B).

In addition, the radiographic results showed that the new drainage plug could help postoperative patients with large wounds return to near complete healing. After approximately 18 months of treatment with the new drainage plug, the cyst cavity healed completely with good new bone formation (Figure 6).

\section{Discussion}

Mandibular cyst is a common disease of the oral and maxillofacial region, and is divided into odontogenic and non-odontogenic cysts. Odontogenic cysts evolve from dental tissue or teeth, while non-odontogenic cysts are derived from epithelium remaining in the jaw during embryonic development. Odontogenic cysts are more common in clinical practice and are mostly benign lesions. Most cystic lesions in the mandible are due to the gradual accumulation of excessive fluid secreted by lining epithelial cells. The hydrostatic pressure of cyst fluid is an important factor affecting the absorption and digestion of cysts by surrounding tissues. Hence, decompression or excision have become the most common methods for conservative treatment of large mandibular cysts. These methods reduce the size of cysts by changing the hydrostatic pressure of cyst fluid, and ultimately reduce the extent of surgical excision or remove the need for surgery (18-20). After decompression, continuous drainage of cyst fluid to release cyst pressure is key in the late stages of conservative treatment (21).

Denture bases and clasps have been reported to make fillers (22). However, the poor plasticity of these materials leads to the irregular shape of fillers, unsmooth boundaries, and lack of a good fit between the filler and the wound, often resulting in discomfort and secondary injury. Moreover, silicone residues of impression materials 

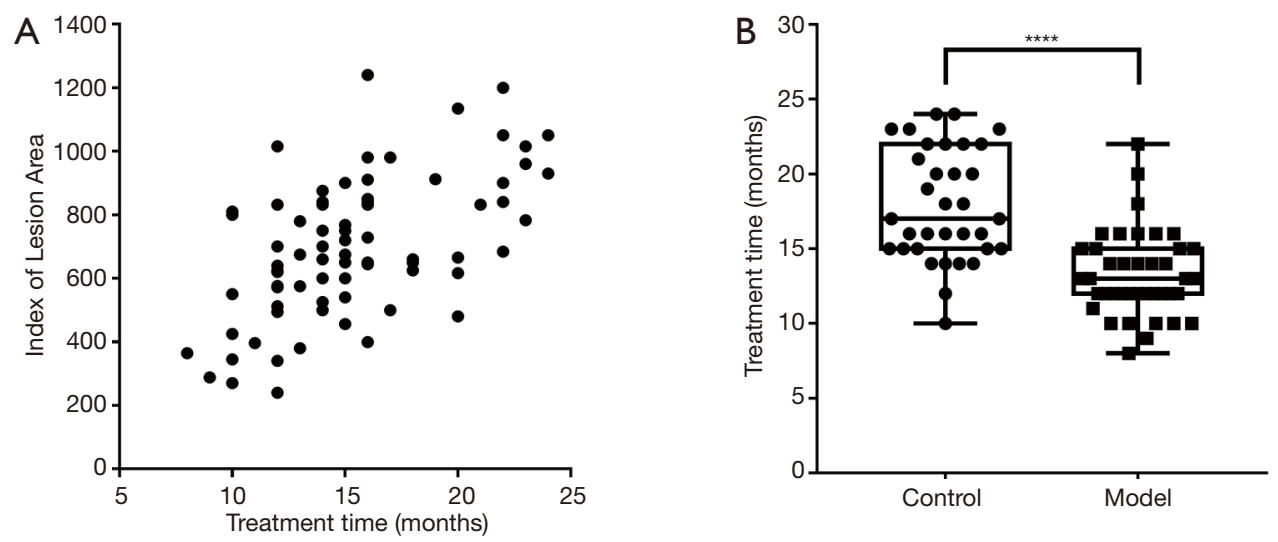

Figure 5 Efficacy evaluation of the new drainage plug. (A) An approximately linear positive correlation between cystic lesion area and treatment time. (B) The new drainage plug (model) significantly shortened treatment time compared with the conventional plug (control). $* * * *, \mathrm{P}<0.0001$.
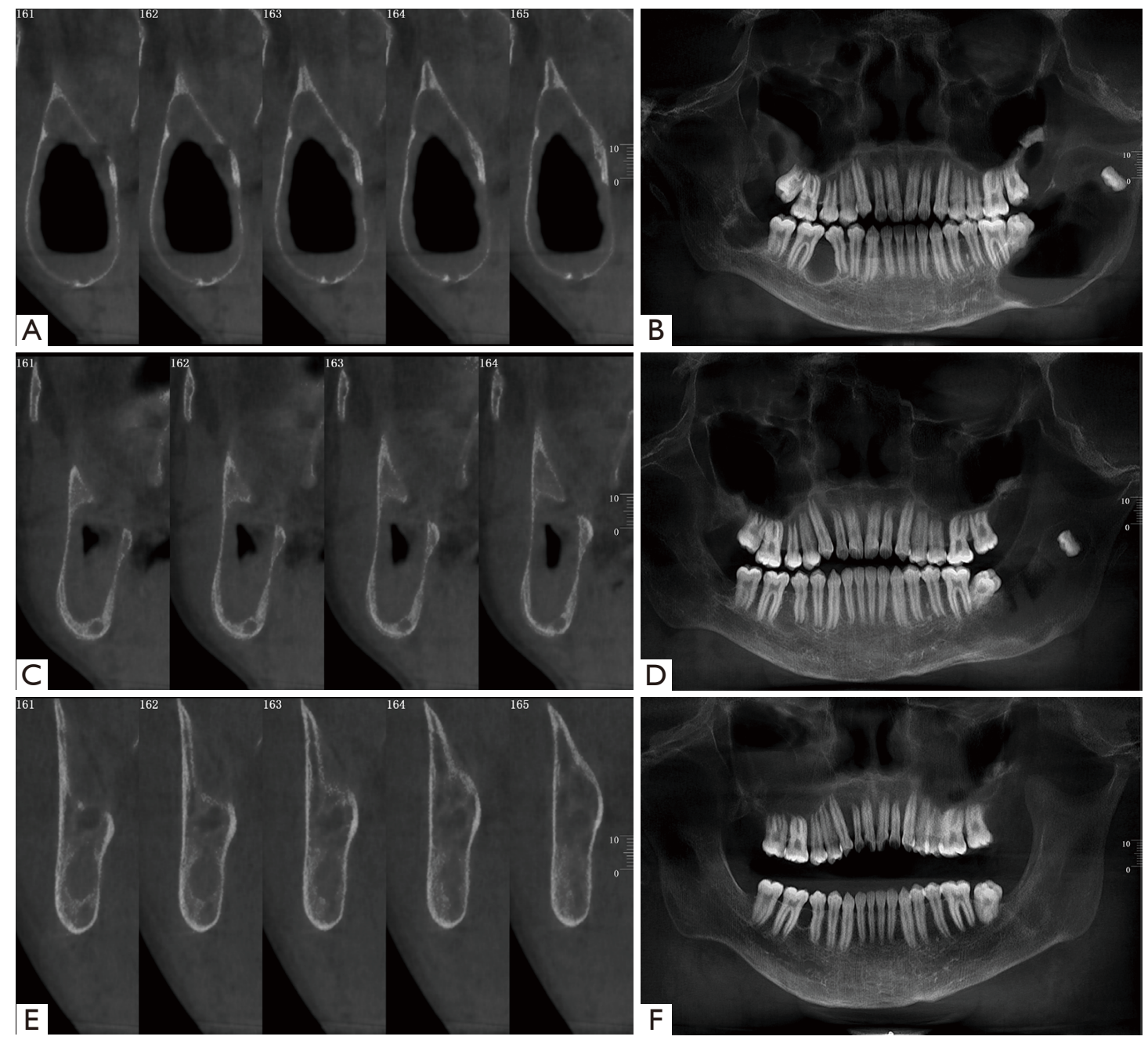

Figure 6 Therapeutic effect at each stage of the drainage plug. (A,B) The CBCT section views of the cystic lesion before treatment. (C,D) The CBCT section views of the cystic lesion after using the drainage plug for 4 months. The wound became significantly smaller. (E,F) The CBCT section views of the cystic lesion after using the drainage plug for 18 months. The wound almost completely healed. 
easily fall and can be left in the cyst cavity, which triggers inflammation and can lead to poor outcomes. In the late stages of rehabilitation, denture base resin filler is inconvenient to remove and can easily cause secondary injury to patients. In addition, a stent placed between the cyst and the oral mucosa has also been confirmed to allow drainage and decompression of the cyst (9). However, food can obstruct the stent repeatedly, leading to stent dislocation and falling. Hence, further surgical intervention is required to remove the stent and reinsert it. This technique also causes other common complications such as local pressure necrosis and tissue scarring.

To improve these problems, we designed a new silicone covered drainage plug, consisting of an acrylic resin frame covered with a thin silicone layer, with a drainage tube in the frame. After suturing on the $10^{\text {th }}$ day after operation, this drainage plug was placed in the postoperative cavity. Patients were also instructed to rinse the gap between the plug and cyst cavity with sterile saline through the tube at least 4 times per day.

Compared with the traditional denture base, the silicone covered drainage plug has some advantages. First, the drainage tube of the drainage plug allows communication between the cyst cavity and the oral cavity, and facilitates timely drainage of the new postoperative cyst effusion to release pressure in the cavity. This minimizes hydrostatic pressure in the cyst, thereby improving new bone formation and shortening recovery time. In addition, the drainage plug fits well with the patient's oral cavity, which effectively prevents deformity in the oral cavity caused by fenestration, and avoids food accumulation and postoperative secondary injuries.

In this new drainage plug, the acrylic resin frame is covered with elastic silicone. The elastic material directly lines the surgical wound to form a tight junction, avoiding the cumbersome operation of setting a fixed hook or occlusal support through the impression procedure and tooth preparation procedure on the abutment teeth. Therefore, the installation steps of the drainage plug are greatly reduced, with better comfort and sealing. Furthermore, due to the reduction of installation devices and steps, the removal of the drainage plug is also convenient. After certain guidance and practice, patients can easily master the process of installation, removal, cleaning, and reinstallation. Compared with the conventional plug, this new drainage plug can effectively shorten the postoperative care time and has a better prognosis. The average wear time is reduced by nearly 5 months.

\section{Conclusions}

This new silicone drainage plug not only facilitates the timely drainage of effusion from the cyst cavity to achieve decompression, but also prevents wound deformity and food accumulation. In addition, it can shorten the clinical recovery time. This plug is of great significance in the treatment of large mandibular cysts and is worthy of further promotion and application.

\section{Acknowledgments}

Funding: None.

\section{Footnote}

Reporting Checklist: The authors have completed the CONSORT reporting checklist. Available at http://dx.doi. org/10.21037/apm-20-2464

Data Sharing Statement: Available at http://dx.doi. org/10.21037/apm-20-2464

Conflicts of Interest: All authors have completed the ICMJE uniform disclosure form (available at http://dx.doi. org/10.21037/apm-20-2464). The authors have no conflicts of interest to declare.

Ethical Statement: The authors are accountable for all aspects of the work in ensuring that questions related to the accuracy or integrity of any part of the work are appropriately investigated and resolved. All procedures performed in this study involving human participants were in accordance with the Declaration of Helsinki (as revised in 2013). This study was approved by the Ethics Committee of the Affiliated Stomatological Hospital of Nanchang University (No. 2019017). All patients volunteered to participate and provided informed consent.

Open Access Statement: This is an Open Access article distributed in accordance with the Creative Commons Attribution-NonCommercial-NoDerivs 4.0 International License (CC BY-NC-ND 4.0), which permits the noncommercial replication and distribution of the article with the strict proviso that no changes or edits are made and the original work is properly cited (including links to both the formal publication through the relevant DOI and the license). See: https://creativecommons.org/licenses/by-nc-nd/4.0/. 


\section{References}

1. Kenney JG, Smoot EC, Morgan RF, et al. Recognizing the temporomandibular joint ganglion. Ann Plast Surg 1987;18:323-6.

2. Guzzo M, Quattrone P, Bianchi R, et al. Dental Invasion by Adenoid Cystic Carcinoma of the Oral Cavity. Ann Maxillofac Surg 2017;7:148-50.

3. Zhao YF, Wei JX, Wang SP. Treatment of odontogenic keratocysts: a follow-up of 255 Chinese patients. Oral Surg Oral Med Oral Pathol Oral Radiol Endod 2002;94:151-6.

4. Segami N, Nojima T. Ganglion cyst in the temporomandibular joint: A case report and discussion of surgical approaches. Cranio 2019;37:400-4.

5. Dongol A, Sagtani A, Jaisani MR, et al. Dentigerous Cystic Changes in the Follicles Associated with Radiographically Normal Impacted Mandibular Third Molars. Int J Dent 2018;2018:2645878.

6. Nakamura N, Mitsuyasu T, Mitsuyasu Y, et al. Marsupialization for odontogenic keratocysts: long-term follow-up analysis of the effects and changes in growth characteristics. Oral Surg Oral Med Oral Pathol Oral Radiol Endod 2002;94:543-53.

7. Zhao Y, Liu B, Han QB, et al. Changes in bone density and cyst volume after marsupialization of mandibular odontogenic keratocysts (keratocystic odontogenic tumors). J Oral Maxillofac Surg 2011;69:1361-6.

8. Saravana GH, Subhashraj K. Cystic changes in dental follicle associated with radiographically normal impacted mandibular third molar. Br J Oral Maxillofac Surg 2008;46:552-3.

9. Giuliani M, Grossi GB, Lajolo C, et al. Conservative management of a large odontogenic keratocyst: report of a case and review of the literature. J Oral Maxillofac Surg 2006;64:308-16.

10. Enislidis G, Fock N, Sulzbacher I, et al. Conservative treatment of large cystic lesions of the mandible: a prospective study of the effect of decompression. Br J Oral Maxillofac Surg 2004;42:546-50.

11. Torres-Lagares D, Segura-Egea JJ, Rodríguez-Caballero A, et al. Treatment of a large maxillary cyst with marsupialization, decompression, surgical endodontic therapy and enucleation. J Can Dent Assoc 2011;77:b87.

12. Gama TM, Dos Santos Brito E, Da Silva LV, et al. CONSERVATIVE TREATMENT OF EXTENSIVE MANDIBULAR MIDLINE CYSTIC LESION-CASE REPORT. Oral Surgery, Oral Medicine, Oral Pathology and Oral Radiology 2020;129:e109.

13. Gao L, Wang XL, Li SM, et al. Decompression as a treatment for odontogenic cystic lesions of the jaw. J Oral Maxillofac Surg 2014;72:327-33.

14. Anavi Y, Gal G, Miron H, et al. Decompression of odontogenic cystic lesions: clinical long-term study of 73 cases. Oral Surg Oral Med Oral Pathol Oral Radiol Endod 2011;112:164-9.

15. Shimamoto H, Chindasombatjaroen J, Kakimoto N, et al. Perineural spread of adenoid cystic carcinoma in the oral and maxillofacial regions: evaluation with contrast-enhanced CT and MRI. Dentomaxillofac Radiol 2012;41:143-51.

16. Shudou H, Sasaki M, Yamashiro T, et al. Marsupialisation for keratocystic odontogenic tumours in the mandible: longitudinal image analysis of tumour size using 3D visualised CT scans. Int J Oral Maxillofac Surg 2012;41:290-6.

17. Y L, W M, Y N, et al. Application of 3D Printed Cyst Plug in Mandibular Cystic Lesions. Journal of Oral Science Research 2019; v.35:52-5.

18. Song IS, Park HS, Seo BM, et al. Effect of decompression on cystic lesions of the mandible: 3-dimensional volumetric analysis. Br J Oral Maxillofac Surg 2015;53:841-8.

19. Yang Z, Liang Q, Yang L, et al. Marsupialization of mandibular cystic ameloblastoma: Retrospective study of 7 years. Head Neck 2018;40:2172-80.

20. Guo GF. Effect of fenestration decompression on odontogenic cyst of mandible and its influence on new bone. The Journal of Medical Theory and Practice 2020.

21. Liang YJ, He WJ, Zheng PB, et al. Inferior alveolar nerve function recovers after decompression of large mandibular cystic lesions. Oral Dis 2015;21:674-8.

22. Tan ZZ, Liu B, Wei JX, et al. Effects of mandibular odontogenic keratocyst surgery and removable partial prostheses on masticatory performance. J Prosthet Dent 2007;97:107-11.

(English Language Editor: C. Betlazar-Maseh)

Cite this article as: Zhang L, Pei J, Huang M, Lian W. Application of a new drainage plug for large mandibular cysts after fenestration decompression. Ann Palliat Med 2021;10(1):590 596. doi: 10.21037/apm-20-2464 